\title{
The problem of singularities and chaos in cosmology
}

\author{
A Yu Kamenshchik
}

Dipartimento di Fisica and Istituto Nazionale di Fisica Nucleare, via Irnerio 46, 40126 Bologna, Italy

L.D. Landau Institute for Theoretical Physics of Russian Academy of Sciences, Kosygin str. 2, 119334 Moscow, Russia

\begin{abstract}
We consider different aspects of the problem of cosmological singularity such as the BKL oscillatory approach to the singularity, the new features of the cosmological dynamics in the neighbourhood of the singularity in multidimensional and superstring cosmological models and their connections with such a modern branch of mathematics as infinite-dimensional Lie algebras. The chaoticity of the oscillatory approach to the cosmological singularity is also discussed. The Conclusion contains some thoughts about the past and the future of the Universe in the light of the oscillatory approach to the Big Bang and the Big Crunch cosmological singularities.
\end{abstract}

\section{Introduction}

Many years ago, in conversations with his students, Lev Davidovich Landau used to say that there were three problems most important for the theoretical physics: the problem of cosmological singularity, the problem of phase transitions and the problem of superconductivity [1]. Now we know that the great breakthrough was achieved in the explanation of phenomena of superconductivity [2] and phase transitions [3. The problem of cosmological singularity was widely studied during the last 50 years and many important results were obtained, but it still conserves some intriguing aspects. Moreover some quite unexpecting facets of the problem of cosmological singularity were discovered. Isaak Marcovich Khalatnikov, who was one of the pupils of L.D. Landau, had brought a significant contribution into the discovery and elaboration of different aspects of the problem of the cosmological singularity and the chaos, arising in the process of the asymptotic approach to this singularity.

In our review, published 10 years ago [4] in the issue of this journal, dedicated to the 90th anniversary of L D Landau birth, we had discussed some questions connected with the problem of singularity in cosmology. In the paper dedicated to 100th anniversary of L D Landau [5] we have dwelled on relations between the well-known old results of these studies and the new developments in this area.

In the present paper, dedicated to 90th anniversary of I.M. Khalatnikov, I shall give a brief review of some old and new ideas, connected with the develop- 
ment of the theory of the asymptotic approach to the cosmological singularity, and shall try to argue why it could be of interest not only for physicists and mathematicians, but, perhaps, for even more wide audience.

To begin with, let us remember that R Penrose and S Hawking [6] proved the impossibility of indefinite continuation of geodesics under certain conditions. This was interpreted as pointing to the existence of a sigularity in the general solution of the Einstein equations. These theorems, however, did not allow for finding the particular analytical structure of the singularity. The analytical behaviour of the general solutions of the Einstein equations in the neighborhood of singularity was investigated in the papers by E M Lifshitz and I M Khalatnikov [7, 8, 9] and V A Belinsky, E M Lifshitz and I M Khalatnikov 10, 11, 12. These papers revealed the enigmatic phenomenon of an oscillatory approach to the singularity which has become known also as the Mixmaster Universe 13. The model of the closed homogeneous but anisotropic universe with three degrees of freedom (Bianchi IX cosmological model) was used to demonstrate that the universe approaches the singularity in such a way that its contraction along two axes is accompanied by an expansion with respect to the third axis, and the axes change their roles according to a rather complicated law which reveals a chaotical behavior [11, 12, 14, 15.

The study of the dynamics of the universe in the vicinity of the cosmological singularity has become an explodingly developing field of modern theoretical and mathematical physics. First of all we would like to mention the generalization of the study of the oscillatory approach to the cosmological singularity in multidimensional cosmological models. It was noticed 16 that the approach to the cosmological singularity in the multidimensional (Kaluza-Klein type) cosmological models has a chaotic character in the spacetimes whose dimensionality is not higher then ten, while in the spacetimes of higher dimensionalities a universe after undergoing a finite number of oscillations enters into a monotonic Kasner-type contracting regime.

The development of cosmological studies based on superstring models has revealed some new aspects of the dynamics in the vicinity of the singularity [18. First, it was shown that in these models exist mechanisms of changing of Kasner epochs provoked not by the gravitational interactions but by the influence of other fields present in these theories. Second, it was proved that the cosmological models based on six main superstring models plus $D=11$ supergravity model exhibit the chaotical oscillatory approach towards the singularity. Third, the connection between cosmological models, manifesting the oscillatory approach towards singularity and a special subclass of infinite-dimensional Lie algebras 19 - the so called hyperbolic Kac-Moody algebras was discovered (a comprehensive review of the corresponding mathematical tools with their application to the BKL studies was given in 20). The study of the algebraic structures, staying behind the chaotical approach to the cosmological singularity opens some new (though still very weakly elaborated) prospectives for the development of the consistent quantum gravity theory [21].

Speaking about the new aspects of the oscillatory approach to the cosmological singularity in the multidimensional and superstring theories, one should not forget that already "classical" BKL behaviour for the $3+1$ dimensional general relativity is not yet totally understood and worth further studying. Besides, I can try to attract attention to some philosophical aspects of this phenomenon which were underestimated until now. 
The structure of the paper is the following: in Sec. 2 we briefly discuss the Landau theorem about the singularity which was not published in a separate paper and was reported in the book [22] and in the review [7]; in Sec. 3 we shall recall the main features of the oscillatory approach to the singularity in relativistic cosmology, including its chaoticity; Sec. 4 will be devoted to the modern development of the BKL ideas and methods, including the dynamics in the presence of a massless scalar field, multidimensional cosmology, superstring cosmology and the correspondence between chaotic cosmological dynamics and hyperbolic Kac-Moody algebras; in the conclusive Sec. 5 we shall express some thoughts about the past and the future of the universe in light of the BKL phenomenon.

\section{Landau theorem about the singularity}

Let us consider the synchronous reference frame with the metric

$$
d s^{2}=d t^{2}-\gamma_{\alpha \beta} d x^{\alpha} d x^{\beta},
$$

where $\gamma_{\alpha \beta}$ is the spatial metric. L D Landau pointed out that the determinant $g$ of the metric tensor in a synchronous reference system must go to zero at some finite time provided some simple conditions on the equation of state are satisfied. To prove this statement it is convenient to write down the $0-0$ component of the Ricci tensor as

$$
R_{0}^{0}=-\frac{1}{2} \frac{\partial K_{\alpha}^{\alpha}}{\partial t}-\frac{1}{4} K_{\alpha}^{\beta} K_{\beta}^{\alpha},
$$

where $K_{\alpha \beta}$ is the extrinsic curvature tensor defined as

$$
K_{\alpha \beta}=\frac{\partial \gamma_{\alpha \beta}}{\partial t}
$$

and the spatial indices are raised and lowered by the spatial metric $\gamma_{\alpha \beta}$. The Einstein equation for $R_{0}^{0}$ is

$$
R_{0}^{0}=T_{0}^{0}-\frac{1}{2} T
$$

where the energy-momentum tensor is

$$
T_{i}^{j}=(\rho+p) u_{i} u^{j}-\delta_{i}^{j} p,
$$

where $\rho, p$ and $u_{i}$ are the energy density, the pressure and the four-velocity respectively. The quantity $T_{0}^{0}-\frac{1}{2} T$ in the right-hand side of equation (4) is

$$
T_{0}^{0}-\frac{1}{2} T=\frac{1}{2}(\rho+3 p)+(\rho+p) u_{\alpha} u^{\alpha},
$$

and is positive provided

$$
\rho+3 p>0
$$

Thus, from Eq. (4) follows that

$$
\frac{1}{2} \frac{\partial K_{\alpha}^{\alpha}}{\partial t}+\frac{1}{4} K_{\alpha}^{\beta} K_{\beta}^{\alpha} \leq 0 .
$$


Because of the algebraic inequality

$$
K_{\alpha}^{\beta} K_{\beta}^{\alpha} \geq \frac{1}{3}\left(K_{\alpha}^{\alpha}\right)^{2}
$$

we have

$$
\frac{\partial K_{\alpha}^{\alpha}}{\partial t}+\frac{1}{6}\left(K_{\alpha}^{\alpha}\right)^{2} \leq 0
$$

or

$$
\frac{\partial}{\partial t} \frac{1}{K_{\alpha}^{\alpha}} \geq \frac{1}{6} .
$$

If $K_{\alpha}^{\alpha}>0$ at some moment of time, then if $t$ decreases, the quantity $\frac{1}{K_{\alpha}^{\alpha}}$ decreases to zero within a finite time. Hence $K_{\alpha}^{\alpha}$ goes to $+\infty$ and that means because of the identity

$$
K_{\alpha}^{\alpha}=\gamma^{\alpha \beta} \frac{\partial g_{\alpha \beta}}{\partial t}=\frac{\partial}{\partial t} \ln g
$$

that the determinant $g$ goes to zero (no faster than $t^{6}$ according to the inequality (11)). If at the initial moment $K_{\alpha}^{\alpha}<$, then the same result will be obtained for the increasing time. The similar result was obtained by Raychaudhuri 23 for the case of dust-like matter and by Komar 24.

This result does not prove that it is inevitable that there exists a true physical singularity, which is one belonging to the spacetime itself and is not connected with the character of the chosen reference system. However, it played an important role stimulating the discussion about the existence and generality of the singularities in cosmology. Notice that the condition of the energodominance (77) used for the proof of the Landau theorem appears also in the proof of the Penrose and Hawking singularity theorem [6]. Moreover, the breakdown of this condition is necessary for an explanation of the phenomenon of cosmic acceleration.

The Landau theorem is deeply connected with the appearance of caustics studied by E M Lifshitz, I M Khalatnikov and V V Sudakov 25 and discussed between them and L D Landau in 1961. Trying to construct geometrically the synchronous reference frame, one start from the three-dimensional Cauchy surface and design the family of geodesics orthogonal to this this surface. The length along these geodesics serves as the time measure. It is known that these geodesics intersect on some two-dimensional caustic surface. This geometry constructed for the empty space is valid also in the presence of dust-like matter $(p=0)$. Such matter, moving along the geodesics concentrates on caustics, but the growth of density cannot be unbounded because the arising pressure destroys the caustics 1 . This question was studied by L P Grishchuk [26. Later V I Arnold, S F Shandarin and Ya B Zeldovich [27] have used the caustics for the explanation of the initial clustering of the dust, which though does not create physical singularities, is nevertheless responsible for the creation of the so called pancakes. These pancakes represent the initial stage of the development of the large scale structure of the universe.

\footnotetext{
${ }^{1}$ In an empty space the caustic is a mathematical, but not a physical singularity. This follows simply from the fact that we can always shift its location by changing the initial Cauchy surface.
} 


\section{Oscillatory approach to the singularity in rel- ativistic cosmology}

One of the first exact solutions found in the framework of general relativity was the Kasner solution [17] for the Bianchi-I cosmological model representing gravitational field in an empty space with Euclidean metric depending on time according to the formula

$$
d s^{2}=d t^{2}-t^{2 p_{1}} d x^{2}-t^{2 p_{2}} d y^{2}-t^{2 p_{3}} d z^{2},
$$

where the exponents $p_{1}, p_{2}$ and $p_{3}$ satisfy the relations

$$
p_{1}+p_{2}+p_{3}=p_{1}^{2}+p_{2}^{2}+p_{3}^{2}=1 .
$$

Remarkably, this solution was the first non-stationary cosmological solution, found before isotropic Friedmann solutions. Perhaps, because of its "exoticity" it was for many years ignored by the working cosmologists and has become appreciated only in the fifties.

Choosing the ordering of exponents as

$$
p_{1}<p_{2}<p_{3}
$$

one can parameterize them as 7

$$
p_{1}=\frac{-u}{1+u+u^{2}}, p_{2}=\frac{1+u}{1+u+u^{2}}, p_{3}=\frac{u(1+u)}{1+u+u^{2}} .
$$

As the parameter $u$ varies in the range $u \geq 1, p_{1}, p_{2}$ and $p_{3}$ assume all their permissible values

$$
-\frac{1}{3} \leq p_{1} \leq 0,0 \leq p_{2} \leq \frac{2}{3}, \frac{2}{3} \leq p_{3} \leq 1 .
$$

The values $u<1$ lead to the same range of values of $p_{1}, p_{2}, p_{3}$ since

$$
p_{1}\left(\frac{1}{u}\right)=p_{1}(u), p_{2}\left(\frac{1}{u}\right)=p_{3}(u), p_{3}\left(\frac{1}{u}\right)=p_{2}(u) .
$$

The parameter $u$ introduced in early sixties has appeared very useful and its properties attract attention of researchers in different contests. For example, in the recent paper [28] a connection was established between the LifshitzKhalatnikov parameter $u$ and the invariants, arising in the context of the Petrov's classification of the Einstein spaces [29.

In the case of Bianchi-VIII or Bianchi-IX cosmological models the Kasner regime (13), (14) ceased to be an exact solution of Einstein equations, however one can design the generalized Kasner solutions [8]-[12]. It is possible to construct some kind of perturbation theory where exact Kasner solution (13), (14) plays role of zero-order approximation while role of perturbations play those terms in Einstein equations which depend on spatial curvature tensors (apparently, such terms are absent in Bianchi-I cosmology). This theory of perturbations is effective in the vicinity of singularity or, in other terms, at $t \rightarrow 0$. The remarkable feature of these perturbations consists in the fact that they imply 
the transition from the Kasner regime with one set of parameters to the Kasner regime with another one.

The metric of the generalized Kasner solution in a synchronous reference system can be written in the form

$$
d s^{2}=d t^{2}-\left(a^{2} l_{\alpha} l_{\beta}+b^{2} m_{\alpha} m_{\beta}+c^{2} n_{\alpha} n_{\beta}\right) d x^{\alpha} d x^{\beta},
$$

where

$$
a=t^{p_{l}}, b=t^{p_{m}}, c=t^{p_{n}} .
$$

The three-dimensional vectors $\vec{l}, \vec{m}, \vec{n}$ define the directions along which the spatial distances vary with time according to the power laws (20). Let $p_{l}=p_{1}, p_{m}=$ $p_{2}, p_{n}=p_{3}$ so that

$$
a \sim t^{p_{1}}, b \sim t^{p_{2}}, c \sim t^{p_{3}},
$$

i.e. the Universe is contracting in directions given by vectors $\vec{m}$ and $\vec{n}$ and is expanding along $\vec{l}$. It was shown [11] that the perturbations caused by spatial curvature terms make the variables $a, b$ and $c$ to undergo transition to another Kasner regime characterized by the following formulae:

$$
a \sim t^{p^{\prime}}, b \sim t^{p_{2}^{\prime}}, c \sim t^{p_{3}^{\prime}},
$$

where

$$
p_{l}^{\prime}=\frac{\left|p_{1}\right|}{1-2\left|p_{1}\right|}, p_{m}^{\prime}=-\frac{2\left|p_{1}\right|-p_{2}}{1-2\left|p_{1}\right|}, p_{n}^{\prime}=-\frac{p_{3}-2\left|p_{1}\right|}{1-2\left|p_{1}\right|} .
$$

Thus, the effect of the perturbation is to replace one "Kasner epoch" by another so that the negative power of $t$ is transformed from the $\vec{l}$ to the $\vec{m}$ direction. During the transition the function $a(t)$ reaches a maximum and $b(t)$ a minimum. Hence, the previously decreasing quantity $b$ now increases, the quantity $a$ decreases and $c(t)$ remains a decreasing function. The previously increasing perturbation caused the transition from regime (21) to that (22) is damped and eventually vanishes. Then other perturbation begins grow which leads to a new replacement of one Kasner epoch by another, etc.

We would like to emphasize that namely the fact that perturbation implies such a change of dynamics which extinguishes it gives us an opportunity to use perturbation theory so successfully. Let us note that the effect of changing of the Kasner regime exists already in the cosmological models more simple than those of Bianchi IX and Bianchi VIII. As a matter of fact in the Bianchi II universe there exists only one type of perturbations, connected with the spatial curvature and this perturbation makes one change of Kasner regime (one bounce). This fact was known to E M Lifshitz and I M Khalatnikov at the beginning of sixties and they have discussed this topic with L D Landau (just before the tragic accident) who has appreciated it highly. The results describing the dynamics of the Bianchi IX model were reported by I M Khalatnikov in his talk given in January 1968 in Henri Poincare Seminar, in Paris. John A Wheeler who was present there pointed out that the dynamics of the Bianchi IX universe represents a non-trivial example of the chaotic dynamical system. Later Kip Thorn has distributed the preprint with the text of this talk.

Coming back to the rules governing the bouncing of the negative power of time from one direction to another it is necessary to emphasize that the very complicated system of non-linear differential equations in partial derivatives 
is reduced in the vicinity of singularity to rather a simple system of ordinary differential equations. To extract the information about the rules (23) it was enough to make a qualitative analysis of the latter. This analysis could be compared with the description of the motion of the ball climbing to the top of a hill: after the reaching of the highest possible point, it stops and begins rolling down. At the foot of the hill its velocity is equal to its initial velocity, but with the opposite sign. Besides, some kind of the law of the conservation of the sum of velocities corresponding to the expansion (contraction) of different space directions was used [11, 22]

On the other hand, it was shown that the rules of bouncing (23) could be conveniently expressed by means of the parametrization (16):

$$
p_{l}=p_{1}(u), p_{m}=p_{2}(u), p_{n}=p_{3}(u)
$$

and then

$$
p_{l}^{\prime}=p_{2}(u-1), p_{m}^{\prime}=p_{1}(u-1), p_{n}^{\prime}=p_{3}(u-1) .
$$

The greater of the two positive powers remains positive.

The successive changes (25), accompanied by a bouncing of the negative power between the directions $\vec{l}$ and $\vec{m}$, continue as long as the integral part of $u$ is not exhausted, i.e. until $u$ becomes less that one. Then, according to Eq. (18) the value $u<1$ transforms into $u>1$, at this moment either the exponent $p_{l}$ or $p_{m}$ is negative and $p_{n}$ becomes smaller one of the two positive numbers $\left(p_{n}=p_{2}\right)$. The next sequence of changes will bounce the negative power between the directions $\vec{n}$ and $\vec{l}$ or $\vec{n}$ and $\vec{m}$. Let us emphasize that the usefulness of the Lifshitz-Khalatnikov parameter $u$ is connected with the circumstance that it allows to encode rather complicated laws of transitions between different Kasner regimes (23) in such simple rules as $u \rightarrow u-1$ and $u \rightarrow \frac{1}{u}$.

Consequently, the evolution of our model towards a singular point consists of successive periods (called eras) in which distances along two axes oscillate and along the third axis decrease monotonically, the volume decreases according to a law which is near to $\sim t$. In the transition from one era to another, the axes along which the distances decrease monotonically are interchanged. The order in which the pairs of axes are interchanged and the order in which eras of different lengths follow each other acquire a stochastic character.

To every ( $s$ th) era corresponds a decreasing sequence of values of the parameter $u$. This sequence has the form $u_{\max }^{(s)}, u_{\max }^{(s)}-1, \ldots, u_{\min }^{(s)}$, where $u_{\min }^{(s)}<1$. Let us introduce the following notation:

$$
u_{\min }^{(s)}=x^{(s)}, u_{\max }^{(s)}=k^{(s)}+x^{(s)}
$$

i.e. $k^{(s)}=\left[u_{\max }^{(s)}\right]$ (the square brackets denote the greatest integer $\leq u_{\max }^{(s)}$ ). The number $k^{(s)}$ defines the era length. For the next era we obtain

$$
u_{\max }^{(s+1)}=\frac{1}{x^{(s)}}, k^{(s+1)}=\left[\frac{1}{x^{(s)}}\right] .
$$

The ordering with respect to the length of $k^{(s)}$ of the successive eras (measured by the number of Kasner epochs contained in them) acquires asymptotically a stochastic character. The random nature of this process arises because 
of the rules (26)-(27) which define the transitions from one era to another in the infinite sequence of values of $u$. If all this infinite sequence begins since some initial value $u_{\max }^{(0)}=k^{(0)}+x^{(0)}$, then the lengths of series $k^{(0)}, k^{(1)}, \ldots$ are numbers included into an expansion of a continuous fraction:

$$
k^{(0)}+x^{(0)}=k^{(0)}+\frac{1}{k^{(1)}+\frac{1}{k^{(2)}+\cdots}} .
$$

We can describe statistically this sequence of eras if we consider instead of a given initial value $u_{\max }^{(0)}=k^{(0)}+x^{(0)}$ a distribution of $x^{0)}$ over the interval $(0,1)$ governed by some probability law 14 . Then we also obtain some distributions of the values of $x^{(s)}$ which terminate every sth series of numbers. It can be shown that with increasing $s$, these distributions tend to a stationary (independent of $s$ ) probability distribution $w(x)$ in which the initial value $x^{(s)}$ is completely "forgotten":

$$
w(x)=\frac{1}{(1+x) \ln 2} .
$$

It follows from Eq. (29) that the probability distribution of the lengths of series $k$ is given by

$$
W(k)=\frac{1}{\ln 2} \ln \frac{(k+1)^{2}}{k(k+2)} .
$$

The source of stochasticity arising at the oscillatory approach to the cosmological singularity can be described in such terms: the transition from one Kasner era to another is described by the transformation of the interval $[0,1]$ into itself by the formula

$$
T x=\left\{\frac{1}{x}\right\}, \text { i.e., } x_{s+1}=\left\{\frac{1}{x_{s}}\right\} .
$$

This transformation is expanding and possesses the property of exponential instability. It is not one to one transformation. Its inverse is not unique. In other words, fixing the value of the parameter $u$ we can predict the evolution towards singularity, but we cannot describe the past.

One can pass over from a one-sided infinite sequence

$$
\left(x_{0}, x_{1}, x_{2}, \ldots\right),
$$

to a doubly infinite sequence [15]

$$
X=\left(\ldots, x_{-2}, x_{-1}, x_{0}, x_{1}, x_{2}, \ldots\right) .
$$

The sequence $X$ is equivalent to a sequence of integers

$$
K=\left(\ldots, k_{-2}, k_{-1}, k_{0}, k_{1}, k_{2}, \ldots\right),
$$

such that

$$
k_{s}=\left[\frac{1}{x_{s-1}}\right]
$$

Inversely,

$$
x_{s}=\frac{1}{k_{s+1}+\frac{1}{k_{s+2}+\cdots}} \equiv x_{s+1}^{+}
$$




$$
x_{s}^{+}=\left[k_{s}, k_{s+1}, \ldots\right]
$$

and

$$
x_{s}^{-} \equiv\left[k_{s-1}, k_{s-2}, \ldots\right]
$$

The shift of entire sequence $X$ to the right means a joint transformation

$$
x_{s+1}^{+}=\left\{\frac{1}{x_{s}^{+}}\right\}, x_{s+1}^{-}=\frac{1}{\left(\left[\frac{1}{x_{s}^{+}}\right]+x_{s}^{-}\right)} .
$$

This is a one-to-one mapping in the unit square, which permits to calculate in an exact manner probability distributions for other parameters describing successive eras such as parameter $\delta$ giving relation between the amplitudes of logarithms of functions $a, b, c$ and logarithmic time [15].

Thus, we have seen from the results of statistical analysis of evolution in the neighbourhood of singularity [14] that the stochasticity and probability distributions of parameters arise already in classical general relativity.

At the end of this section a historical remark is in order. The continuous fraction (28) was shown in 1968 to I M Lifshitz (L D Landau has already left) and he immediately noticed that one can derive the formula for a stationary distribution of the value of $x(29)$. Later it becomes known that this formula was derived in nineteenth century by Karl F Gauss, who had not published it but had described it in a letter to one of colleagues.

\section{Oscillatory approach to the singularity: mod- ern development}

The oscillatory approach to the cosmological singularity described in the preceding section was developed for the case of an empty spacetime. It is not difficult to understand that if one considers the universe filled with a perfect fluid with the equation of state $p=w \rho$, where $p$ is the pressure, $\rho$ is the energy density and $w<1$, then the presence of this matter cannot change the dynamics in the vicinity of the singularity. Indeed, using the energy conservation equation one can show that

$$
\rho=\frac{\rho_{0}}{(a b c)^{w+1}}=\frac{\rho_{0}}{t^{w+1}}
$$

where $\rho_{0}$ is a positive constant. Thus, the term representing the matter in the Einstein equations behaves like $\sim 1 / t^{1+w}$ and at $t \rightarrow 0$ is weaker than the terms of geometrical origin coming from the time derivatives of the metric, which behaves like $1 / t^{2}$, let alone the perturbations due to the presence of spatial curvature, responsible for changes of a Kasner regime,which behave like $1 / t^{2+4\left|p_{1}\right|}$. However, the situation changes drastically, if the parameter $w$ is equal to one, i.e. the pressure is equal to the energy density. Such kind of matter is called "stiff matter" and can be represented by a massless scalar field. In this case $\rho \sim 1 / t^{2}$ and the contribution of matter is of the same order as main terms of geometrical origin. Hence, it is necessary to find a Kasner type solution, taking into account the presence of terms, connected with the presence of the stiff matter (a massless scalar field). Such study was carried out in paper 
30. It was shown that again the scale factors $a, b$ and $c$ can be represented as $t^{2 p_{1}}, t^{2 p_{2}}$ and $t^{2 p_{3}}$ respectively, where the Kasner indices satisfy the relations:

$$
p_{1}+p_{2}+p_{3}=1, \quad p_{1}^{2}+p_{2}^{2}+p_{3}^{2}=1-q^{2}
$$

where the number $q^{2}$ reflects the presence of the stiff matter and is bounded by

$$
q^{2} \leq \frac{2}{3}
$$

One can see that if $q^{2}>0$, then exist combinations of the positive Kasner indices, satisfying the relations (41). Moreover, if $q^{2} \geq \frac{1}{2}$ only sets of three positive Kasner indices can satisfy the relations (41). If a universe finds itself in a Kasner regime with three positive indices, the perturbative terms, existing due to the spatial curvatures are too week to change this Kasner regime, and thus, it becomes stable. That means, that in the presence of the stiff matter, the universe after a finite number of changes of Kasner regimes finds itself in a stable regime and oscillations stop. Thus, the massless scalar field plays "anti-chaotizing" role in the process of the cosmological evolution [30]. One can use the Lifshitz-Khalatnikov parameter also in this case. The Kasner indices satisfying the relations (41) are conveniently represented as [30]

$$
\begin{aligned}
p_{1} & =\frac{-u}{1+u+u^{2}}, \\
p_{2} & =\frac{1+u}{1+u+u^{2}}\left[u-\frac{u-1}{2}\left(1-\left(1-\beta^{2}\right)^{1 / 2}\right)\right], \\
p_{3} & =\frac{1+u}{1+u+u^{2}}\left[1+\frac{u-1}{2}\left(1-\left(1-\beta^{2}\right)^{1 / 2}\right)\right], \\
\beta^{2} & =\frac{2\left(1+u+u^{2}\right)^{2}}{\left(u^{2}-1\right)^{2}} .
\end{aligned}
$$

The range of the parameter $u$ now is $-1 \leq u \leq 1$, while the admissible values of the parameter $q$ at some given $u$ are

$$
q^{2} \leq \frac{\left(u^{2}-1\right)^{2}}{2\left(1+u+u^{2}\right)^{2}} .
$$

One can easily show that after one bounce the value of the parameter $q^{2}$ changes according to the rule

$$
q^{2} \rightarrow q^{2}=q^{2} \times \frac{1}{\left(1+2 p_{1}\right)^{2}}>q^{2} .
$$

Thus, the value of the parameter $q^{2}$ grows and, hence, the probability to find all the three Kasner indices to be positive increases. It confirms again the statement that after a finite number of bounces the universe in the presence of the massless scalar field finds itself in the Kasner regime with three positive indices and the oscillations stop.

In the second half of eighties a series of papers was published [16], where were studied the solutions of Einstein equations in the vicinity of singularity for $d+1$-dimensional spacetimes. The multidimensional analog of a Bianchi-I 
universe was considered, where the metric is a generalized Kasner metric:

$$
d s^{2}=d t^{2}-\sum_{i=1}^{d} t^{2 p_{i}} d x^{i 2},
$$

where the Kasner indices $p_{i}$ satisfy the conditions

$$
\sum_{i=1}^{d} p_{i}=\sum_{i=1}^{d} p_{i}^{2}=1
$$

In the presence of spatial curvature terms the transition from one Kasner epoch to another occurs and this transition is described by the following rule: the new Kasner exponents are equal to

$$
p_{1}^{\prime}, p_{2}^{\prime}, \ldots, p_{d}^{\prime}=\text { ordering of }\left(q_{1}, q_{2}, \ldots, q_{d}\right),
$$

with

$$
\begin{aligned}
& q_{1}=\frac{-p_{1}-P}{1+2 p_{1}+P}, q_{2}=\frac{p_{2}}{1+2 p_{1}+P}, \ldots, \\
& q_{d-2}=\frac{p_{d-2}}{1+2 p_{1}+P}, q_{d-1}=\frac{2 p_{1}+P+p_{d-1}}{1+2 p_{1}+P}, \\
& q_{d}=\frac{2 p_{1}+P+p_{d}}{1+2 p_{1}+P}
\end{aligned}
$$

where

$$
P=\sum_{i=2}^{d-2} p_{i} .
$$

However, such a transition from one Kasner epoch to another occurs if at least one of the numbers $\alpha_{i j k}$ is negative. These numbers are defined as

$$
\alpha_{i j k} \equiv 2 p_{i}+\sum_{l \neq j, k, i} p_{l},(i \neq j, i \neq k, j \neq k) .
$$

For the spacetimes with $d<10$ one of the factors $\alpha$ is always negative and, hence one change of Kasner regime is followed by another one, implying in such a way the oscillatory behaviour of the universe in the neighbourhood of the cosmological singularity. However, for the spacetimes with $d \geq 10$ exist such combinations of Kasner indices, satisfying Eq. (47) and for which all the numbers $\alpha_{i j k}$ are positive. If a universe enters into the Kasner regime with such indices, ( so called "Kasner stability region") its chaotical behaviour disappears and this Kasner regime conserves itself. Thus, the hypothesis was forward that in the spacetimes with $d \geq 10$ after a finite number of oscillations the universe under consideration finds itself in the Kasner stability region and the oscillating regime is replaced by the monotonic Kasner behaviour.

The discovery of the fact that the chaotic character of the approach to the cosmological singularity disappears in the spacetimes with $d \geq 10$ was unexpected and looked as an accidental result of a game between real numbers satisfying the generalized Kasner relations (49). Later it becomes clear that behind this fact there is a deep mathematical structure, namely, the hyperbolic KacMoody algebras. Indeed, in the series of works by Damour, Henneaux, Nicolai 
and some other authors (see e.g. Refs. 18) on the cosmological dynamics in the models based on superstring theories, living in 10-dimensional spacetime and on the $d+1=11$ supergravity model, it was shown that in the vicinity of the singularity these models reveal oscillating behaviour of the BKL type. The important new feature of the dynamics in these models is the role played by non-gravitational bosonic fields ( $p$-forms) which are also responsible for transitions from one Kasner regime to another. For description of these transitions the Hamiltonian formalism 13 becomes very convenient. In the framework of such formalism the configuration space of the Kasner parameters describing the dynamics of the universe could be treated as a billiard while the curvature terms in Einstein theory and also $p$-form's potentials in superstring theories play role of walls of these billiards. The transition from one Kasner epoch to another is the reflection from one of the walls. Thus, there is a correspondence between rather complicated dynamics of a universe in the vicinity of the cosmological singularity and the motion of an imaginary ball on the billiard table.

However, there exists a more striking and unexpected correspondence between the chaotical behaviour of the universe in the vicinity of the singularity and such an abstract mathematical object as the hyperbolic Kac-Moody algebras 18. Let us explain briefly what does it mean. Every Lie algebra is defined by its generators $h_{i}, e_{i}, f_{i}, i=1, \ldots, r$, where $r$ is the rank of the Lie algebra, i.e. the maximal number of its generators $h_{i}$ which commutes each other (these generators constitute the Cartan subalgebra). The commutation relations between generators are

$$
\begin{aligned}
& {\left[e_{i}, f_{j}\right]=\delta_{i j} h_{i},} \\
& {\left[h_{i}, e_{j}\right]=A_{i j}=A_{i j} e_{j},} \\
& {\left[h_{i}, f_{j}\right]=-A_{i j} f_{j},} \\
& {\left[h_{i}, h_{j}\right]=0 .}
\end{aligned}
$$

The coefficients $A_{i j}$ constitute the generalized Cartan $r \times r$ matrix such that $A_{i i}=2$, its off-diagonal elements are non-positive integers and $A_{i j}=0$ for $i \neq j$ implies $A_{j i}=0$. One can say that the $e_{i}$ are rising operators, similar to well-known operator $L_{+}=L_{x}+i L_{y}$ in the theory of angular momentum, while $f_{i}$ are lowering operators like $L_{-}=L_{x}-i L_{y}$. The generators $h_{i}$ of the Cartan subalgebra could be compared with the operator $L_{z}$. The generators should also obey the Serre's relations

$$
\begin{aligned}
& \left(\operatorname{ad} e_{i}\right)^{1-A_{i j}} e_{j}=0, \\
& \left(\operatorname{ad} f_{i}\right)^{1-A_{i j}} f_{j}=0,
\end{aligned}
$$

where $(\operatorname{ad} A) B \equiv[A, B]$.

The Lie algebras $\mathcal{G}(A)$ build on a symmetrizable Cartan matrix $A$ have been classified according to properties of their eigenvalues:

if $A$ is positive definite, $\mathcal{G}(A)$ is a finite-dimensional Lie algebra;

if $A$ admits one null eigenvalue and the others are all strictly positive $\mathcal{G}(A)$ is an Affine Kac-Moody algebra;

if $A$ admits one negative eigenvalue and all the others are strictly positive, $\mathcal{G}(A)$ is a Lorentz KM algebra.

There exists a correspondence between the structure of a Lie algebra and a certain system of vectors in the $r$-dimensional Euclidean space, which simplify 
essentially the task of classification of the Lie algebras. These vectors called roots represent the rising and lowering operators of the Lie algebra. The vectors corresponding to the generators $e_{i}$ and $f_{i}$ are called simple roots. The system of simple positive roots (i.e. the roots, corresponding to the rising generators $e_{i}$ ) can be represented by nodes of their Dynkin diagrams, while the edges connecting (or non connecting) the nodes give an information about the angles between simple positive root vectors.

An important subclass of Lorentz KM algebras can be defined as follows: A KM algebra such that the deletion of one node from its Dynkin diagram gives a sum of finite or affine algebras is called an hyperbolic KM algebra. These algebras are all known. In particular, there exists no hyperbolic algebra with rank higher than 10 .

Let us recall some more definitions from the theory of Lie algebras. The reflections with respect to hyperplanes orthogonal to simple roots leave the systems of roots invariant. The corresponding finite-dimensional group is called Weyl group. Finally, the hyperplanes mentioned above divide the $r$-dimensional Euclidean space into regions called Weyl chambers. The Weyl group transform one Weyl chamber into another.

Now, we can briefly formulate the results of approach [18 following the paper [31: the links between the billiards describing the evolution of the universe in the neighbourhood of singularity and its corresponding Kac-Moody algebra can be described as follows:

the Kasner indices describing the "free" motion of the universe between the reflections from the wall correspond to the elements of the Cartan subalgebra of the KM algebra;

the dominant walls, i.e. the terms in the equations of motion responsible for the transition from one Kasner epoch to another, correspond to the simple roots of the KM algebra;

the group of reflections in the cosmological billiard is the Weyl group of the KM algebra;

the billiard table can be identified with the Weyl chamber of the KM algebra.

One can imagine two types of billiard tables: infinite such where the linear motion without collisions with walls is possible (non-chaotic regime) and those where reflections from walls are inevitable and the regime can be only chaotic. Remarkably, the Weyl chambers of the hyperbolic KM algebras are designed in such a way that infinite repeating collisions with walls occur. It was shown that all the theories with the oscillating approach to the singularity such as Einstein theory in dimensions $d<10$ and superstring cosmological models correspond to hyperbolic KM algebras.

The existence of links between the BKL approach to the singularities and the structure of some infinite-dimensional Lie algebras has inspired some authors to declare a new program of development of quantum gravity and cosmology 21]. They propose "to take seriously the idea that near the singularity (i.e. when the curvature gets larger than the Planck scale) the description of a spatial continuum and space-time based (quantum) field theory breaks down, and should be replaced by a much more abstract Lie algebraic description. Thereby the information previously encoded in the spatial variation of the geometry and of the matter fields gets transferred to an infinite tower of Lie-algebraic variables depending only on "time". in other words we are led to the conclusion 
that space-and thus, upon quantization also space-time - actually disappears (or "de-emerges") as the singularity is approached."

\section{Conclusion: some thoughts about the past and the future of the universe}

In the preceding section we have told briefly about the newest development of the theory of the BKL approach to the cosmological singularity connected with the superstring-inspired cosmological models and the infinite-dimensional Lie algebras. However, already in the "standard" $3+1$ - dimensional general relativity the effect of the oscillatory approach to the singularity and the chaoticity implied by it is of great interest. Indeed, the discovery of the non-static timedependent cosmological solutions in the general relativity, first of all the Friedmann solutions, has given birth to the animate discussions on such questions as:

Did the universe have the beginning ?

Will the universe have the end ?

Can the Universe exist during a finite interval of time?

What was before the beginning and what will be after the end ?

These questions look quite reasonable, because we know that in all three Friedmann models - flat, open and closed - the universe has the beginning and this beginning is nothing but the Big Bang singularity. In the closed Friedmann model the universe has also the end - the Big Crunch singularity and exists during a finite period of time. Moreover, according to the so called Standard Cosmological Model, based on a rather large set of observational data, something like Big Bang has taken place approximately 13.7 billions years ago (measured in terms of the cosmic, i.e. synchronous time). The more or less accepted existence of the beginning of the evolution of the universe and the possible existence of the end of the universe can be source of the joy for those who believe into the creation of the universe and for whom its possible end can also be confirmation of their philosophical or theological beliefs. It is curious that the Pontifical Academy of Sciences has organized a special conference in the October-November of 2008 in Vatican with the title "Scientific insights into the evolution of the universe and of life". On the other hand, the possibility of the finite-time existence of the universe can provoke some kind of psychological discomfort in persons whom the finite duration of the existence of the universe seems to be senseless. For somebody, the fact that its own existence takes place in the universe which lives only during a finite period of time, can look depressive.

Analyzing this aspects of the problem of the evolution of the universe, one should ask himself/herself, which time parametrization should we use, speaking about the time of the existence of the Universe ? As we know since the epoch of the creation of the special relativity, the time is relative. In the framework of the general relativity the time becomes even more relativity and can run with different rates at different spatial points. Making conformal transformations (for example, then one constructs the Penrose conformal diagrams [6]) one can turn an infinite time interval into the finite time interval. Thus, why should we use the cosmic time? The answer on this question is simple: the cosmic 
time for a particle staying in rest in a Friedmann homogeneous and isotropic universe coincides with the proper time, introduced in the special relativity. Hence, when we are considering the present day universe it is quite reasonable to discuss it in terms of the cosmic time and to say that the universe was created 13.7 billions years ago. However, when we consider the vicinity of the Big Bang cosmological singularity in the past, or when we accept the possibility of existence of the future Big Crunch singularity in the distant future, the situation changes drastically. The universe is extremally anisotropic in the neighbourhood of such singularities and is described by the chaotic succession of Kasner epochs and eras, as was discussed above. (One can make a precision here: while choosing very special isotropic initial conditions one can avoid the chaoticity in the neighbourhood of the Big Bang singularity, which can , in principle have the Friedmannian form, it is impossible not to have the chaotic regime in the vicinity of the Big Crunch singularity, because, the inhomogeneities, developed during the evolution of the universe, make its contracting stage highly anistropic [32]).

Thus, while the evolution between an arbitrary moment of cosmic time and the moment corresponding to the initial Big Bang or final Big Crunch singularity occupies a finite interval of cosmic time, an infinite number of events occurs during this finite period. The infinite chaotic succession of Kasner epochs and eras, makes senseless the cosmic time as a measure of a cosmological evolution. Indeed, we have an infinite history that separates us from the birth of the Universe at the Big Bang. If the contraction of the universe culminating in the encounter with the Big Crunch singularity waits for us in the future [33, we still have an infinite number of events in front of us. Thus, the BKL oscillatory regime of approaching to the cosmological singularity screens us from the Big Bang and Big Crunch.

From the mathematical point of view it means, that in the vicinity of singularity the natural time parameter is not the cosmic time, but the logarithmic time. Then, while the cosmic time runs from the moment zero, corresponding to the singularity to some finite moment $t_{1}$, the logarithmic time runs from $-\infty$ to $\ln t_{1}$, spanning an infinite interval of time.

Remarkably, the comment concerning the importance of the logarithmic time can be found already in the last before last paragraph of the Landau and Lifshitz monograph 22]: "The successive series of oscillations crowd together as we approach the singularity. An infinite number of oscillations are contained between any finite world time $t$ and the moment $t=0$. The natural variable for describing the time behaviour of this evolution is not the time $t$, but its logarithm, $\ln t$, in terms of which the whole process of approach to the singular point is spread out to $-\infty$ ".

The similar idea is expressed also in the cited earlier paper by Damour and Nicolai [21]: "There is no "quantum bounce" bridging the gap between an incoming collapsing and an outgoing expanding quasi-classical universe. Instead "life continues" at the singularity for an infinite affine time,but with the understanding that (i) dynamics no longer "takes place" in space, and (ii) the infinite affine time[measured, say, by the Zeno-like time coordinate $t$ ] corresponds to a sub-Planckian interval $0<T<T_{\text {Planck }}$ of geometrical proper time." Curiously, the analog of the object that the authors of 21] call Zeno-like time is the so called spatial tortoise coordinate in the Schwarzschild geometry [34]. Both these names have their origin in the Zeno's paradox about Achilles and the tortoise, 
which is, perhaps, the first example of the transforming of the finite time interval into an infinite one (see, e.g. the section I of the third part of the third volume of the "War and Peace" by Leo Tolstoj 35]).

Concluding, I would like to say semiseriously that the discovery of the oscillatory approach to the cosmological singularity has a practical meaning: it liberates us from the fear of the End of the World.

This work was partially supported by the RFBR grant 08-02-00923 and by the grant LSS-4899.2008.2.

\section{References}

[1] Landau L D, Private communication

[2] Bardeen J, Cooper L N, Schrieffer J R Phys. Rev. 1081175 (1957)

[3] Wilson K G, Kogut J "The renormalization group and the $\varepsilon$-expansion" Phys. Rep. 1275 (1974) [Translated into Russian (Moscow: Mir, 1975)]

[4] Khalatnikov I M, Kamenshchik A Yu Usp. Fiz. Nauk 168593 (1998) [Physics-Uspekhi $4 \mathbf{1} 525$ (1998)]

[5] Khalatnikov I M, Kamenshchik A Yu Usp. Fiz. Nauk 178639 (2008) [Physics-Uspekhi 51609 (2008)]

[6] Penrose R Structure of Space-Time (New York, Amsterdam:W.A. Benjamin, 1968) [Translated into Russian (Moscow:Mir, 1972)]; Hawking S W, Ellis G F R The Large Scale Structure of Space-Time (Cambridge, New York: Cambridge Unive. Press, 1973) [Translated into Russian (Moscow:Mir, (1977)]; Hawking S W, Penrose R Proc. R. Soc. London Ser. A 314529 (1970)

[7] Lifshitz E M, Khalatnikov I M Usp. Fiz. Nauk 80391 (1963) [Sov. Phys. Usp. 6495 (1964)]; Adv. Phys. 12185 (1963)

[8] Khalatnikov I M, Lifshitz E M Phys. Rev. Lett. 2476 (1970)

[9] Lifshitz E M, Khalatnikov I M Pis'ma Zh. Eksp. Teor. Fiz. 11200 (1970) [JETP Lett. 11123 (1970)]

[10] Belinsky V A, Khalatnikov I M Zh. Eksp. Teor. Fiz. 561700 (1969) [Sov. Phys. JETP 29911 (1969)

[11] Belinsky V A, Lifshitz E M, Khalatnikov I M Usp. Fiz. Nauk 102463 (1970) [Sov. Phys. Usp. 13745 (1971)]

[12] Belinsky V A, Lifshitz E M, Khalatnikov I M Adv. Phys. 31639 (1982)

[13] Misner C W Phys. Rev. Lett 221071 (1969)

[14] Lifshitz E M, Lifshitz I M, Khalatnikov I M Zh. Eksp. Teor. Fiz 59322 (1970) [Sov. Phys. JETP 32173 (1971)]

[15] Khalatnikov I M, Lifshitz E M, Khanin K M, Shchur L N, Sinai Ya G J. Stat. Phys. 3897 (1985) 
[16] Demaret J, Hanquin J-L, Henneaux M, Spindel P Phys. Lett. B 16427 (1985); Demaret J, Henneaux M, Spindel P, Taormina A Phys. Lett. B 175 129 (1986); Demaret J, de Rop Y, Henneaux M Int. J. Theor. Phys. 28 1067 (1989)

[17] Kasner E M Amer. J. Math. 43217 (1921)

[18] Damour T and Henneaux M Phys. Rev. Lett. 85920 (2000); Damour T, Henneaux M, Julia B, Nicolai H Phys. Lett. B 509323 (2001); Damour T, Henneaux M, Nicolai H Class. Quantum Grav. 20 R145 (2003)

[19] Kac V G Infinite dimensional Lie algebras (Cambridge: Cambridge University Press, 1990)

[20] Henneaux M, Persson D and Spindel P, Living Rev. Rel. 111 (2008)

[21] Damour T, Nicolai H Int. J. Mod. Phys. D 17525 (2008)

[22] Landau L D, Lifshitz E M Teoriya Polya (The Classical Theory of Fields) ( Moscow: Nauka, 1988) [Translated into English (Oxford: Pergamon Press, 1975)]

[23] Raychaudhuri R Phys. Rev. 981123 (1955); Phys. Rev. 106172 (1957)

[24] Komar A Phys. Rev. 104544 (1956)

[25] Lifshitz E M, Sudakov V V, Khalatnikov I M ZhETF 401847 (1961) [Sov. Phys. JETP 131298 (1961)]; Phys. Rev. Lett. 6311 (1961)

[26] Grishchuk L P ZhETF 51475 (1966) [Sov. Phys. JETP 24320 (1967)]

[27] Arnold V I, Shandarin S F, Zeldovich Ya B Geophys. Astrophys. Fluid Dynamics 20111 (1982)

[28] Bini D, Cherubini C, Jantzen R T Class. Quantum Grav. 245627 (2007)

[29] Petrov A Z Prostranstva Einsteina (Einstein spaces) (Moscow, Izd. Fiz. Mat. Lit.] [Translated into English (Oxford: Pergamon Press, 1969)]

[30] Belinsky V A, Khalatnikov I M Zh. Eksp. Teor. Fiz. 631121 (1972) [Sov. Phys. JETP 36591 (1973)]

[31] Damour T Cosmological Singularities, Billiards and Lorentzian Kac-Moody algebras gr-qc/0412105 (2004)

[32] Belinski V Cosmological singularity arXiv:0910.0374 [gr-qc] (2009)

[33] Kallosh R, Linde A D, Prokushkin S, Shmakova M Phys. Rev. D 66123503 (2002); Alam U, Sahni V, Starobinsky A A JCAP 0304002 (2003)

[34] Misner C W, Thorne K S, Wheeler J A Gravitation (San Francisco: W.H. Freeman and Company, 1973)

[35] Tolstoj L N Vojna i Mir (Moscow: Eksmo, 2008) [translated into English (London: Penguin Books, 1978)] 